\title{
The association of CREBRF variant rs373863828 with body composition in adult Samoans
}

\author{
Nicola L. Hawley ${ }^{1 *}$, Rachel L. Duckham ${ }^{2},{ }^{3 *}$, Jenna C. Carlson ${ }^{4,11}$, Take Naseri ${ }^{5}$,
} Muagututia Sefuiva Reupena ${ }^{6}$, Viali Lameko ${ }^{7}$, Alysa Pomer $^{1}$, Abigail Wetzel ${ }^{8}$, Melania Selu $^{9}$, Vaimoana Lupematisila ${ }^{9}$, Folla Unasa ${ }^{9}$, Lupesina Vesi ${ }^{9}$, Tracy Fatu ${ }^{9}$, Seipepa Unasa $^{9}$, Kima Faasalele-Savusa ${ }^{9}$, Satupaitea Viali ${ }^{10}$, Anna C. Rivara ${ }^{1}$, Emily M. Russell $^{11}$, Ranjan Deka ${ }^{12}$, Erin E. Kershaw ${ }^{13 \#}$, Ryan L. Minster ${ }^{11 \#}$, Daniel E. Weeks ${ }^{4,11 \#,}$ Stephen T. McGarvey"\#

*These authors contributed equally to this work \#These authors share senior authorship

\section{Affiliations:}

${ }^{1}$ Department of Chronic Disease Epidemiology, Yale School of Public Health, New Haven, CT, USA

${ }^{2}$ Institute of Physical Activity and Nutrition, Deakin University, Geelong, VIC, Australia ${ }^{3}$ Australian Institute for Musculoskeletal Science (AIMSS), The University of Melbourne and Western Health, St Albans, Victoria, Australia ${ }^{4}$ Department of Biostatistics, Graduate School of Public Health, University of Pittsburgh, Pittsburgh, PA, USA

${ }^{5}$ Ministry of Health, Apia, Samoa

${ }^{6}$ Lutia I Puava Ae Mapu I Fagalele, Apia, Samoa

${ }^{7}$ Oceania University of Medicine, Apia, Samoa

8International Health Institute, Department of Epidemiology, School of Public Health, Brown University, Providence, RI, USA

${ }^{9}$ Obesity, Lifestyle and Genetic Adaptations Study Group, Apia, Samoa

${ }^{10}$ School of Medicine, National University of Samoa, Apia, Samoa

${ }^{11}$ Department of Human Genetics, Graduate School of Public Health, University of Pittsburgh, Pittsburgh, PA, USA

${ }^{12}$ Department of Environmental and Public Health Sciences, College of Medicine, University of Cincinnati, Cincinnati, OH, USA

${ }^{13}$ Division of Endocrinology, Department of Medicine, University of Pittsburgh, Pittsburgh, PA, USA

Corresponding Author: Nicola L. Hawley Yale School of Public Health 60 College Street New Haven, CT 06510, USA Email: nicola.hawley@yale.edu 


\section{Abstract}

46 Objective: The minor allele of rs373863828, a missense variant in CREBRF, is

47 associated with higher BMI but lower odds of type 2 diabetes in Pacific Islanders.

48 Methods: To test if this protective effect operates through metabolically favorable body

49 fat distribution, we examined the association of the minor A allele with body

50 composition, measured using dual-energy x-ray absorptiometry (DXA), in a cross-

51 sectional study of $n=421$ Samoan adults.

52 Results: We replicated our earlier finding that this allele was associated with higher

53 weight and BMI, although it was statistically significant only in women. There was no

54 significant association of genotype with percent body fat, visceral adiposity or fat

55 distribution, although in women, the A allele was associated with greater total fat mass

$56(p=0.02)$, android $(p=0.009)$ and trunk fat $(p=0.01)$. In both sexes, age- and height-

57 adjusted average lean mass was significantly greater per copy of the A allele: 2.16

$58 \mathrm{~kg} / \mathrm{copy}$ in women and $1.73 \mathrm{~kg} /$ copy in men.

59 Conclusions: These data do not support a primary role of fat distribution in mediating

60 the association between rs373863828 genotype and type 2 diabetes risk. We suggest

61 an alternative hypothesis: those with the A allele may more efficiently regulate blood

62 glucose because of their greater absolute lean mass.

63

64

65

66

67 


\section{Introduction}

69 In 2016, based on a genome-wide association study (GWAS) of body mass index (BMI)

70 in adult Samoans, we reported the identification of a missense variant in CREB3

71 regulatory factor $(C R E B R F)$ that is paradoxically associated with higher $\mathrm{BMI}$ and odds

72 of obesity but with lower fasting blood glucose and odds of type 2 diabetes [1]. The

73 minor allele A of rs373863828, an arginine-to-glutamine missense variant, had a

74 frequency of 0.259 in the discovery sample, indicating that $>40 \%$ of Samoans have at

75 least one copy of the risk allele.

77 Our discovery has been replicated in several other Pacific Islander populations (Māori,

78 Tongans, Cook Islanders, Niueans, Chamorro, Chuukese) [2-6] and may partially

79 explain their greater risk of obesity compared to other ethnic groups. The paradoxical

80 associations of rs373863828 with BMI and diabetes, however, remains unexplained. In

81 a murine 3T3L1 adipocyte model, ectopic expression of the human variant enhanced

82 adipogenesis and lipid storage compared to control [1]. Therefore, we hypothesized that

83 human carriers of the variant would have greater fat mass relative to lean mass but

84 would store that fat in a more metabolically favorable distribution (e.g., subcutaneously

85 rather than viscerally, or peripherally rather than centrally) than those without the

86 variant, thereby explaining their lower odds of diabetes.

87

88 To test that hypothesis, we examined the association between rs373863828 genotype

89 and body composition, measured using dual-energy $x$-ray absorptiometry (DXA).

90 


\section{Methods}

92 Between August 2017 and March 2019 participants from the original GWAS sample

93 were recruited into a follow-up study to examine body composition and cardiometabolic

94 health. Participants with AA, AG, and GG genotypes were targeted in a 1:2:2 ratio.

95 Protocols for the original GWAS in 2010 and the follow-up study, including genotyping

96 methods, have been previously published $[7,8]$.

98 Eligible participants were not pregnant, lactating, or attempting to control their weight

99 through medication or surgery, were resident on the island of 'Upolu and were part of a

100 maximally unrelated sample [maximum kinship 6.01\%] [8]. Willing participants $(n=519$;

101 aged 30.7-72.7 years) gave written informed consent. Protocols were approved by the

102 Yale University Institutional Review Board (IRB \#1604017547), the University of

103 Pittsburgh (\#PRO16040077), and the Health Research Committee of the Samoan

104 Ministry of Health.

106 Weight and height were measured using a Tanita HD 351 digital weighing scale (Tanita

107 Corporation of America, IL) and SECA 213 portable stadiometer (Seca GmbH \& Co.,

108 Germany), respectively. DXA outcomes (assessed using a Lunar iDXA, GE Healthcare

109 Medicine, Encore Version 17) included total body fat, lean, and bone mass. Visceral fat

110 mass (estimated using GE CoreScan ${ }^{\mathrm{TM}}$ ) was also measured along with fat mass in the

111 android (central) and gynoid (hip and thigh) regions and the limbs. Android-to-gynoid fat

112 ratio (a measure of abdominal vs. gluteal fat distribution) was calculated, as well as

113 trunk-to-peripheral fat ratio (TPFR), a measure of centrality of fat deposition (fat mass in 
114 the trunk region divided by the sum of arm and leg fat [peripheral fat]). When

115 participant's width exceeded the scan area, a right-side scan was 'mirrored' and used to

116 estimate total body composition [9]. Contraindications to DXA included exposure to

117 additional X-rays or computed tomography (CT) in the prior 12 months. Therefore, this

118 analysis was restricted to $n=421$ participants (AA $n=72$, AG $n=161, \mathrm{GG} n=188$ ). For

119 analyses of visceral fat mass, the sample was further reduced by $n=1$ participant whose

120 body size prevented the capture of the core region in a single scan.

122 Associations between body size and composition outcomes and rs373863828 genotype

123 were performed using linear models that were sex-stratified and adjusted for age and

$124 \mathrm{age}^{2}$ (sex and age were self-reported by participants). Body composition outcomes

125 were also adjusted for height [10]. Genotype was modeled additively (the number of $A$

126 alleles a participant carries), consistent with previous work [1]. Analyses were

127 conducted in R Version 3.6.0 (R Foundation for Statistical Computing, Austria) with the

128 threshold for statistical significance set at $p<0.05$. For ease of interpretation, effect sizes

129 are presented on their original scale; sensitivity analyses using inverse-normally

130 transformed traits gave similar results.

131

132 Results

133 The rs373863828 minor A allele was associated with higher average weight and BMI,

134 significantly so among women, but not men (Table 1$)$. Average total fat mass $(p=0.02)$,

135 android $(p=0.009)$, and trunk fat mass $(p=0.01)$ were significantly higher with each copy

136 of the minor allele among women but not men (Table 1, Figure 1), although the same 
137 trend toward higher fat mass was present in men. In both sexes, average lean mass

138 was greater with each copy of the minor allele: by $2.16 \mathrm{~kg} / \mathrm{copy}$ in women $(p=0.0001)$

139 and $1.73 \mathrm{~kg} / \mathrm{copy}$ in men $(p=0.02)$ after a6-7 (tdjusting for age, age ${ }^{2}$, and height. There

140 were no significant differences by genotype observed among either sex in visceral

141 adiposity, percent body fat, nor in the distribution of body fat based on android-to-gynoid

142 ratio and TPFR (Table 1). Additional plots describing distribution of body size and

143 composition outcomes by genotype are provided in Supplementary Figure 1.

\section{Discussion}

146 In this study we demonstrate that the original association between the rs373863828

147 minor A allele and BMI persisted after 8 years. Moreover, we observed that the positive

148 effect of the allele on BMI is primarily a function of greater lean mass rather than fat

149 mass among men, and a combination of greater lean and fat mass among women. The

150 findings do not support the hypothesis that the inverse association between the $A$ allele

151 and type 2 diabetes is mediated by more metabolically favorable fat distribution. They

152 are, however, consistent with our recent work in infants where we observed greater lean

153 mass at four months of age [15] among those with the A allele. While it is not possible to

154 obtain the same measures of fat distribution measures in infants, we saw no effect on

155 total or percent fat mass.

156

157 An alternative hypothesis, consistent with these data, is that those with the A allele may

158 more effectively regulate blood glucose as a result of their greater lean mass. Greater

159 muscle and bone mass promote lower serum glucose, greater insulin sensitivity, and 
160 lower risk of diabetes via multiple mechanisms $[11,12]$. Skeletal muscle, in particular, is

161 a major site for insulin-dependent and independent glucose uptake and disposal as well

162 as secretion of autocrine, paracrine, and endocrine factors that influence metabolic

163 homeostasis, such as irisin, interleukin-6, and other myokines $[13,14]$. While outside the

164 scope of this report, we will test this hypothesis in future work by using structural

165 equation modeling to examine the effect of rs373863828 on fasting glucose and the

166 odds of type 2 diabetes as mediated through its direct and indirect effects on lean and

167 fat mass. Further research, using CT or magnetic resonance imaging (MRI) to

168 distinguish between muscle and other lean tissue, which DXA cannot do, will be needed

169 to fully test this new hypothesis.

171 While the minor allele was associated with greater lean mass in both men and women,

172 and height in men (consistent with our earlier work [16]), we observed a stronger effect

173 of the missense variant on BMI in women and an effect on fat mass in women only.

174 Prior studies of this variant and its association with BMI have adjusted for sex, rather

175 than presenting sex-stratified analyses (likely because of their limited sample size) [2-6],

176 so this phenomenon has not been noted previously. Our findings indicate that attempts

177 to examine potential sex-specific effects of the variant are warranted.

179 The major strength of this study compared to other examinations of the rs373863828

180 genotype to date is the use of DXA-measured body composition, rather than BMI, which

181 is unable to distinguish between fat and lean mass. We did, however, encounter many

182 participants (19\% of our recruited sample) who, because of our conservative safety 
183 criteria, could not participate due to recent high dose radiation exposure. While those

184 who could not receive a DXA scan were similar in age, BMI, and genotype distribution,

185 this may limit generalizability of our findings. Furthermore, we note that the participants

186 in our study sample had high average levels of body fat (approximately $30 \%$ in men and

$18745 \%$ in women). Future studies are needed to explore the functional relationship

188 between fat distribution and rs373863828 across the spectrum of adiposity, including

189 examining the impact upon cardiometabolic disease risk factors.

191 With further study of insulin-glucose metabolism and its association with the

192 rs373863828 variant ongoing among several Pacific Islander groups, these findings

193 indicate that greater lean mass among those with the minor allele may be responsible

194 for the partially protective effect of the minor A allele on odds of diabetes. As diabetes

195 prevalence continues to increase among Samoans and other Pacific Islander

196 populations, additional understanding of the mechanisms that underlie this association

197 may be transformative for prevention and treatment.

199 Funding

200 This work was supported by US National Institutes of Health (NIH) National Heart Lung

201 and Blood Institute grant R01 HL093093 (PI: STM). ACR was supported by the US NIH

202 Fogarty International Center Global Health Equity Scholars Program (D43TW010540).

203 The funding bodies had no role in the design or conduct of the study, data analysis, or

204 the decision to submit this manuscript for publication. 
medRxiv preprint doi: https://doi.org/10.1101/2021.02.11.21251582; this version posted February 12, 2021. The copyright holder for this preprint

(which was not certified by peer review) is the author/funder, who has granted medRxiv a license to display the preprint in perpetuity.

All rights reserved. No reuse allowed without permission.

\section{Disclosure}

207 The authors declared no conflict of interest.

208 


\section{References}

1. Minster RL, Hawley NL, Su CT, Sun G, Kershaw EE, Cheng H, et al. A thrifty variant in CREBRF strongly influences body mass index in Samoans. Nat Genet 2016 Sep; 48(9): 1049-1054.

2. Naka I, Furusawa T, Kimura R, Natsuhara K, Yamauchi T, Nakazawa M, et al. A missense variant, rs373863828-A (p.Arg457GIn), of CREBRF and body mass index in Oceanic populations. J Hum Genet 2017 Sep; 62(9): 847-849.

3. Berry SD, Walker CG, Ly K, Snell RG, Atatoa Carr PE, Bandara D, et al. Widespread prevalence of a CREBRF variant amongst Māori and Pacific children is associated with weight and height in early childhood. Int J Obes (Lond) 2018 Apr; 42(4): 603-607.

4. Krishnan M, Major TJ, Topless RK, Dewes O, Yu L, Thompson JMD, et al. Discordant association of the CREBRF rs373863828 A allele with increased BMI and protection from type 2 diabetes in Māori and Pacific (Polynesian) people living in Aotearoa/New Zealand. Diabetologia 2018 Jul; 61(7): 1603-1613.

5. Ohashi J, Naka I, Furusawa T, Kimura R, Natsuhara K, Yamauchi T, et al. Association study of CREBRF missense variant (rs373863828: G>A; p.Arg457Gln) with levels of serum lipid profile in the Pacific populations. Ann Hum Biol 2018 May; 45 (3): 215-219.

6. Hanson RL, Safabakhsh S, Curtis JM, Hsueh W-C, Jones LI, Afalgue TF, et al. Association of CREBRF variants with obesity and diabetes in Pacific Islanders from Guam and Saipan. Diabetologia 2019 Sep; 62(9): 1647-1652.

7. Hawley NL, Minster RL, Weeks DE, Viali S, Reupena MS, Sun G, et al. Prevalence of adiposity and associated cardiometabolic risk factors in the Samoan genome-wide association study. Am J Hum Biol 2014 Jul; 26(4): 491501.

8. Hawley NL, Pomer A, Rivara AC, Rosenthal SL, Duckham RL, Carlson JC, et al. Exploring the paradoxical relationship of a CREBRF missense variant with body mass index and diabetes among Samoans: study protocol for the Soifua Manuia ('Good Health') observational cohort study. JMIR Research Protocols 2020 (in press)

9. Rothney MP, Brychta RJ, Schaefer EV, Chen KY, Skarulis MC. Body composition measured by dual-energy X-ray absorptiometry half-body scans in obese adults. Obesity (Silver Spring) 2009; 17(6): 1281-1286.

10. Shepherd J, Ng B, Sommer M, Heymsfield SB. Body composition by DXA. Bone 2017; 104:101-105.

11. Hong S, Chang Y, Jung H, Yun KE, Shin H, Ryu S. Relative muscle mass and the risk of incident type 2 diabetes: A cohort study. PLoS One 2017;12(11):e0188650. 
12. Bassett DRJ. Skeletal muscle characteristics: relationships to cardiovascular risk factors. Med Sci Sports Exerc 1994;26(8):957-66.

13. Eckel J. Myokines in metabolic homeostasis and diabetes. Diabetologia 2019;62(9):1523-8.

14. Karsenty G, Ferron M. The contribution of bone to whole-organism physiology. Nature 2012;481:314-20.

15. Arslanian KA, Fidow UT, Atanoa T, Unasa-Apelu F, Naseri T, Wetzel Al, Pomer A, Duckham RL, McGarvey ST, Strayer JA, Kershaw EE, Hawley NL. A missense variant in CREBRF, rs373863828, is associated with fat-free mass, not fat mass in Samoan infants. Int J Obes (in press)

16. Carlson JC, Rosenthal SL, Russell EM, Hawley NL, Sun G, Cheng H, Naseri T, Reupena MS, Tuitele J, Deka R, McGarvey ST, Weeks DE, Minster RL. A missense variant in CREBRF is associated with taller stature in Samoans. Am J Hum Biol 2020 [online ahead of print; doi: 10.1002/ajhb.23414] 
Table 1: Age, body size, and body composition by genotype

\begin{tabular}{|c|c|c|c|c|c|c|c|c|c|c|}
\hline & \multicolumn{5}{|c|}{ Female } & \multicolumn{5}{|c|}{ Male } \\
\hline & $\begin{array}{c}\mathbf{G G} \\
(n=103)\end{array}$ & $\begin{array}{c}\text { AG } \\
(n=92)\end{array}$ & $\begin{array}{c}\text { AA } \\
(n=38)\end{array}$ & $\beta$ & $p$ & $\begin{array}{c}\mathbf{G G} \\
(n=85)\end{array}$ & $\begin{array}{c}A G \\
(n=69)\end{array}$ & $\begin{array}{c}\text { AA } \\
(n=34)\end{array}$ & $\beta$ & $p$ \\
\hline Age (years) & $50.8(9.5)$ & $50.6(9.8)$ & $50.0(9.0)$ & -0.36 & 0.67 & $53.1(10.3)$ & $53.7(9.3)$ & $49.0(11.0)$ & -1.59 & 0.11 \\
\hline Body Mass Index $\left(\mathrm{kg} / \mathrm{m}^{2}\right)^{a}$ & $35.8(6.4)$ & $37.1(6.2)$ & $40.3(9.2)$ & 1.95 & 0.0014 & $32.7(5.7)$ & $33.2(5.7)$ & $34.0(5.9)$ & 0.60 & 0.32 \\
\hline Weight $(\mathrm{kg})^{\mathrm{a}}$ & $94.2(19.3)$ & $97.3(17.5)$ & $106.5(24.9)$ & 5.21 & 0.002 & $95.7(21.2)$ & 99.4 (18.1) & $103.8(19.8)$ & 3.67 & 0.06 \\
\hline Height $(\mathrm{cm})^{a}$ & $161.8(5.6)$ & $161.8(5.3)$ & $162.6(6.6)$ & 0.24 & 0.62 & $170.9(6.4)$ & $173.0(6.0)$ & $174.8(5.5)$ & 1.71 & 0.003 \\
\hline Total Fat Mass $(\mathrm{kg})^{\mathrm{b}}$ & $40.9(12.6)$ & $42.6(11.1)$ & $47.0(15.8)$ & 2.51 & 0.02 & $29.1(12.7)$ & $29.8(11.7)$ & $30.3(13.0)$ & -0.11 & 0.93 \\
\hline Total Lean Mass $(\mathrm{kg})^{\mathrm{b}}$ & $50.3(7.4)$ & $51.7(7.5)$ & $55.7(8.3)$ & 2.16 & 0.0001 & $63.2(9.4)$ & $66.2(8.2)$ & $70.1(8.0)$ & 1.73 & 0.02 \\
\hline Total Bone Mass $(\mathrm{kg})^{\mathrm{b}}$ & $2.5(0.4)$ & $2.5(0.4)$ & $2.7(0.4)$ & 0.03 & 0.23 & $3.3(0.5)$ & $3.4(0.4)$ & $3.5(0.4)$ & 0.02 & 0.51 \\
\hline Visceral Fat Mass $(\mathrm{kg})^{\mathrm{b}}$ & $1.33(0.6)$ & $1.47(0.6)$ & $1.49(0.6)$ & 0.09 & 0.10 & $1.9(1.1)$ & $2.0(1.1)$ & $1.8(1.1)$ & -0.01 & 0.96 \\
\hline Missing & 0 & 0 & 0 & & & 0 & 1 & 0 & & \\
\hline Percent Fat $(\%)^{b}$ & $42.8(6.0)$ & $43.5(5.0)$ & $43.6(5.7)$ & 0.46 & 0.36 & $29.2(7.3)$ & $29.1(7.1)$ & $28.1(7.5)$ & -0.38 & 0.60 \\
\hline Android Fat Mass $(\mathrm{kg})^{\mathrm{b}}$ & $3.7(1.3)$ & $3.9(1.2)$ & $4.4(1.6)$ & 0.31 & 0.009 & $3.0(1.6)$ & $3.1(1.5)$ & $3.0(1.6)$ & -0.03 & 0.83 \\
\hline Gynoid Fat Mass $(\mathrm{kg})^{\mathrm{b}}$ & $6.1(2.1)$ & $6.3(1.8)$ & $6.9(2.7)$ & 0.30 & 0.09 & $4.2(1.9)$ & $4.2(1.8)$ & $4.4(1.9)$ & -0.07 & 0.70 \\
\hline Android:Gynoid Fat Ratiob & $0.6(0.1)$ & $0.6(0.1)$ & $0.7(0.1)$ & 0.02 & 0.07 & $0.7(0.2)$ & $0.7(0.2)$ & $0.7(0.2)$ & 0.02 & 0.34 \\
\hline Trunk Fat Mass $(\mathrm{kg})^{\mathrm{b}}$ & $21.7(6.6)$ & $22.8(6.0)$ & $25.1(8.3)$ & 1.50 & 0.01 & $16.8(8.0)$ & $17.5(7.1)$ & $17.1(7.7)$ & -0.11 & 0.88 \\
\hline Peripheral Fat Mass $(\mathrm{kg})^{\mathrm{b}, \mathrm{c}}$ & $18.2(6.3)$ & $18.7(5.4)$ & $20.7(7.9)$ & 0.97 & 0.07 & $11.2(4.8)$ & $11.2(4.7)$ & $12.0(5.3)$ & -0.02 & 0.97 \\
\hline Trunk-to-Peripheral Fat Ratiob & $1.2(0.2)$ & $1.2(0.2)$ & $1.3(0.3)$ & 0.02 & 0.34 & $1.5(0.3)$ & $1.6(0.3)$ & $1.4(0.3)$ & 0.01 & 0.62 \\
\hline
\end{tabular}

Values are means (standard deviation). Beta values are the estimated difference in mean per copy of the minor (A) allele (between AA and AG or AG and GG) from a linear model.

aLinear models were adjusted for age and age $^{2}$

bLinear models were adjusted for age, age ${ }^{2}$, and height

cPeripheral fat mass was calculated from the sum of limb (arm and leg) mass 
medRxiv preprint doi: https://doi.org/10.1101/2021.02.11.21251582; this version posted February 12, 2021. The copyright holder for this preprint (which was not certified by peer review) is the author/funder, who has granted medRxiv a license to display the preprint in perpetuity. All rights reserved. No reuse allowed without permission.

Figure 1: Fat, lean, and bone mass and percent body fat by genotype Total Fat Mass

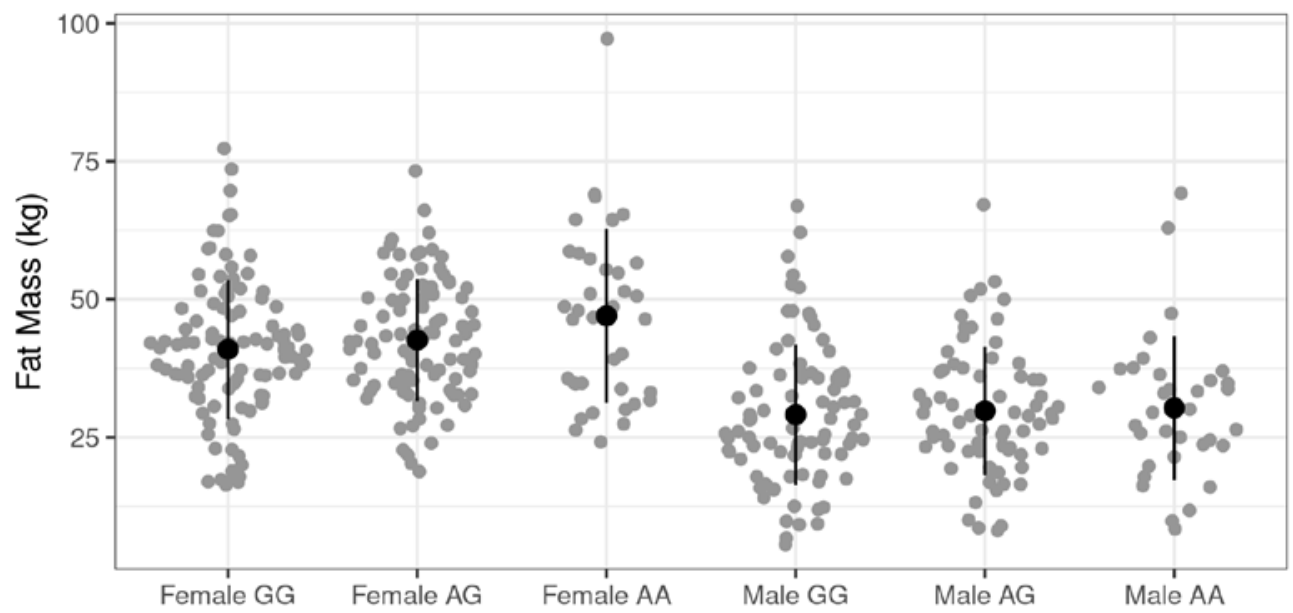

\section{Total Lean Mass}

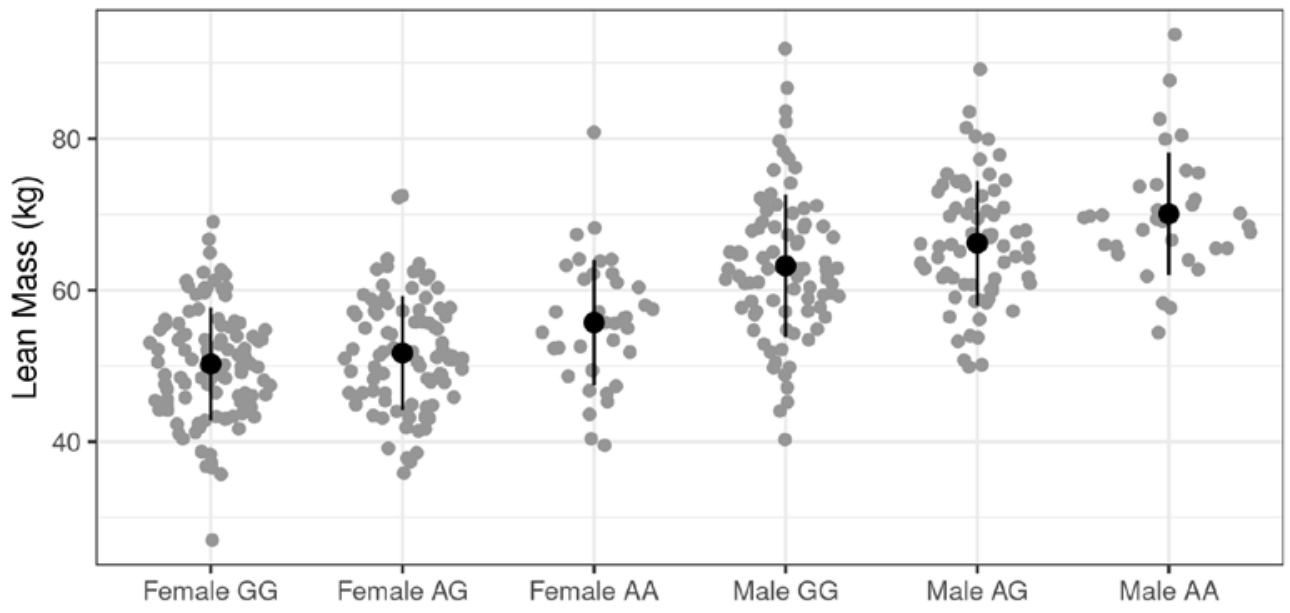

Percent Body Fat

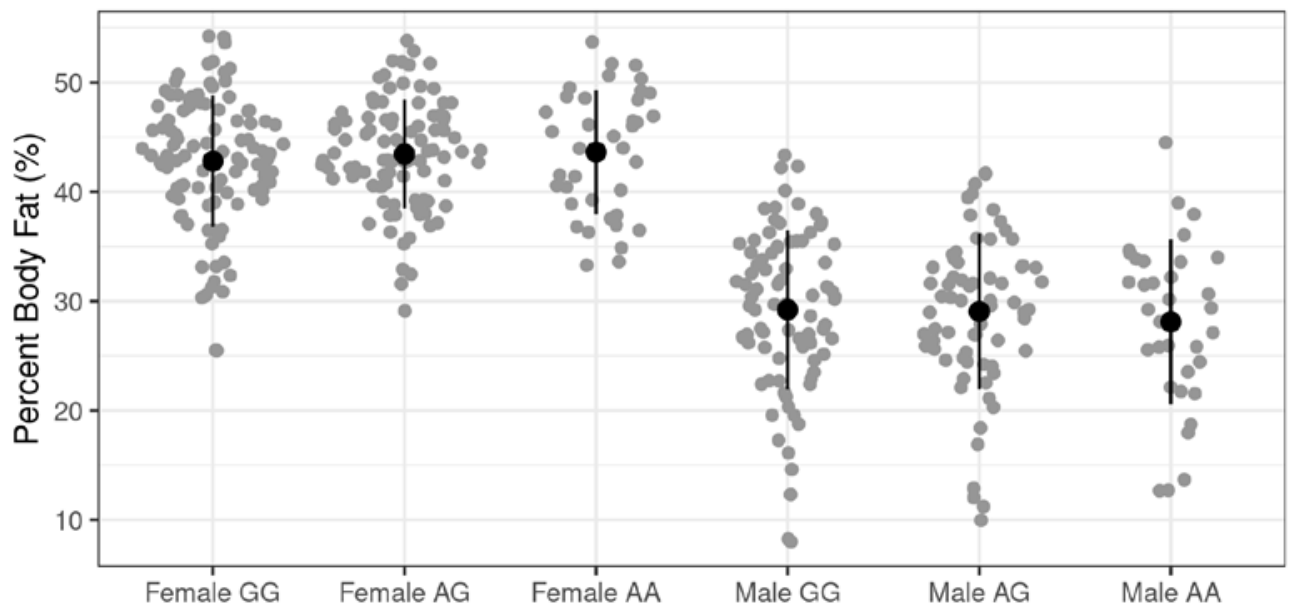

